Article

\title{
Grains Contribute Shortfall Nutrients and Nutrient Density to Older US Adults: Data from the National Health and Nutrition Examination Survey, 2011-2014
}

\author{
Yanni Papanikolaou ${ }^{1, *}$ and Victor L. Fulgoni III 2 (1) \\ 1 Nutritional Strategies, 59 Marriott Place, Paris, ON N3L 0A3, Canada \\ 2 Nutrition Impact, 9725 D Drive North, Battle Creek, MI 49014, USA; vic3rd@aol.com \\ * Correspondence: papanikolaou.yanni@gmail.com; Tel.: +1-519-504-9252
}

Received: 27 March 2018; Accepted: 17 April 2018; Published: 25 April 2018

\begin{abstract}
Previous data demonstrate grain foods contribute shortfall nutrients to the diet of U.S. adults. The 2015-2020 Dietary Guidelines for Americans have identified several shortfall nutrients in the U.S. population, including fiber, folate, and iron (women only). Intake of some shortfall nutrients can be even lower in older adults. The present analyses determined the contribution of grain foods for energy and nutrients in older U.S. adults and ranked to all other food sources in the American diet. Analyses of grain food sources were conducted using a 24-hour recall in adults ( $\geq 51$ years old; $n=4522$ ) using data from the National Health and Nutrition Examination Survey, 2011-2014. All grains provided $278 \mathrm{kcal} /$ day or $14 \%$ of all energy in the total diet, ranking as the 4th largest contributor of energy compared to 15 main food groups. All grain foods ranked 1st for thiamin (33\%) and niacin (23\%) intake relative to 15 main food groups. The grain foods category ranked 2nd highest of 15 main food groups for daily dietary fiber (23\%), iron (38\%), folate ( $40 \%)$, and magnesium (15\%) and was the 3rd largest food group contributor for daily calcium intake $(13 \%)$. When considering nutrients to limit as outlined by dietary guidance, main group of grains contributed $6 \%$ total fat, $5 \%$ saturated fat, $14 \%$ sodium and $9 \%$ added sugar. Breads, rolls and tortillas provided $150 \mathrm{kcal} /$ day or $8 \%$ of all energy in the total diet, ranking as the 2nd largest contributor of energy compared to 46 food subcategories. Breads, rolls and tortillas ranked 1st of 46 foods for daily thiamin $(16 \%)$ and niacin $(10 \%)$ intake and 2 nd for dietary fiber $(12 \%)$, iron $(12 \%)$, folate $(13 \%)$, and magnesium (7\%). Breads, rolls and tortillas ranked 3rd largest food group contributor for daily calcium (5\%) intake. Ready-to-eat cereals provided $47 \mathrm{kcal} /$ day or $2 \%$ of all energy in the total diet, ranking as the 20th largest contributor of energy compared to 46 food subcategories. All ready-to-eat cereals ranked 1 st for daily iron (19\%), 1st for folate (21\%), 5th for dietary fiber $(7 \%)$, 3rd for niacin $(9 \%)$, 8 th for magnesium ( $4 \%)$, and 13th for calcium $(2 \%)$ intake. Given all grain foods and specific subcategories of grain foods provided a greater percentage of several underconsumed nutrients than calories (including dietary fiber, iron, and folate), grain foods provide nutrient density in the American diet of the older adult.
\end{abstract}

Keywords: NHANES; nutrients; aging; grains; fiber

\section{Introduction}

Food sources of energy and nutrients research in the United States (U.S.) has shown that the grain category can play an important contributory role in the diet of all children, adolescents and adults [1,2]. Specifically, grain foods, relative to energy ( $\mathrm{kcal}$ ) provided a greater percentage of 2015-2020 Dietary Guidelines for Americans (2015-2020 DGA) under-consumed nutrients and nutrients of public health concern [3], including dietary fiber, folate, magnesium, calcium and iron. Breads, rolls, tortillas and 
ready-to-eat cereals, as subcategories of grain foods, are also meaningful contributors (i.e., $\geq 10 \%$ in the diet) of dietary fiber, thiamin, folate, iron, zinc and niacin to the American diet [1,2]. Further, earlier data from the National Health and Nutrition Examination Survey (NHANES) 2003-2006 in Americans aged $\geq 2$ years old, identified that significant amounts of vitamins A, B6, B12, C and D, as well as thiamin, riboflavin, niacin, folate and iron were from enriched and/or fortified foods with researchers concluding that without enrichment and/or fortification, nutrient shortfalls are further exacerbated [4]. Nevertheless, previous studies have focused on children and all adults ( $\geq 19$ years old) with no current data isolating older Americans (i.e., >50 years old). Indeed, this demographic represents a significant segment of the U.S. population with the Centers for Disease Control and Prevention (CDC) stating "the growth in the number and proportion of older adults is unprecedented in the history of the U.S. with two factors contributing to the increased number of older adults-increased life spans and aging baby boomers". The CDC has estimated that the American population for those aged 65 years or older will double in 25 years to approximately 72 million [5]. The 2015-2020 Dietary Guidelines for Americans (2015-2020 DGA) recommends all individuals two years of age and older meet nutrient requirements through foods as part of a nutrient-dense diet; however, many individuals fall short in several nutrients, resulting in the declaration that several nutrients are under-consumed relative to the estimated average requirement (EAR; the average daily nutrient intake level estimated to meet the requirements of half of the healthy individuals in a group) or adequate intake levels established by the National Academy of Medicine (formerly known as the Institute of Medicine) [3].

Indeed, healthy aging is multifactorial, however, nutrition is one of the key determinants of successful aging, as it has been documented to promote health and functionality [6]. Thus, adequate intake of macronutrients and micronutrients represents a key factor in healthy aging. The 2015-2020 DGA identified under-consumed nutrients as shortfall nutrients and included vitamins A, D, E, C, folate, calcium, magnesium, fiber, potassium and iron in specific populations [3]. Similar to the preceding policy and scientific advisory committee reports [7,8], the 2015-2020 DGA continues to highlight calcium, potassium, vitamin D and fiber as shortfall 'nutrients of public health concern' in Americans, since under-consumption of these nutrients has been associated with adverse health outcomes. Dietary recommendations further advocate for increased whole grain consumption (i.e., make half your grains whole grains) while limiting enriched/refined grains in the diet [3]. While certain grain food products contribute nutrients to limit in the diet, including added sugar, sodium, and saturated fat, grain foods as a category, including enriched/refined grains also provide a range of beneficial nutrients in the older population, including dietary fiber, calcium, magnesium and B vitamins (thiamin, riboflavin, niacin, cobalamin (B12) and folate) $[1,2,9,10]$. Similar to all of Americans, fiber is a shortfall nutrient and nutrient of public health concern in older adults, where only $4 \%$ of men and $13 \%$ of women had a dietary fiber intake above the recommended Adequate Intake (AI) level [11]. The Dietary Guidelines Scientific Advisory Committee has highlighted that calcium intake from foods and beverages did not meet the EAR for older adults, where $71 \%$ of men and $81 \%$ of females aged $\geq 71$ years old had intakes below the EAR. Similarly, dietary folate and magnesium are underconsumed relative to the Estimated Average Requirement (EAR) in the American population [11]. An analysis using data from the National Health and Nutrition Examination Survey (NHANES) 2009-2012 found that percent of adults below the Estimated Average Requirement (EAR) increased with age for calcium, folate, magnesium, vitamin E and vitamin D [12].

As data in all children and adults support grain food contributions to nutrient density in U.S. dietary patterns and since several nutrients in grains play a distinct role in healthy aging, the objective of the present analyses was to determine sources of energy and nutrients in all grains, and in commonly consumed grain foods (i.e., breads, ready-to-eat cereals, etc.) and rank relative to all other foods in U.S. older adults ( $\geq 51$ years old) using data from NHANES, 2011-2014. 


\section{Experimental Section}

The NHANES is a nationally-representative, cross-sectional survey of U.S. non-institutionalized, civilian residents. NHANES data are collected by the National Center for Health Statistics of the Centers for Disease Control and Prevention. Written informed consent was obtained for all participants or proxies, and the survey protocol was approved by the Research Ethics Review Board at the National Center for Health Statistics. Data from two NHANES datasets (2011-2012; 2013-2014) were combined for the present analyses in adults $\geq 51$ years old [13]. Nutrient intake data for NHANES 2011-2014 are from the United States Department of Agriculture (USDA) Food and Nutrient Database for Dietary Studies (FNDDS) 5.0 and 6.0 [14]. FNDDS are databases that provide the nutrient values for foods and beverages reported in What We Eat in America (WWEIA), the dietary intake component of NHANES for each data release. The WWEIA Food Categories provide an application to analyze food and beverages as consumed in the American diet. The classification scheme includes 150 unique categories and there are 15 main food groups and 46 subcategories of foods. WWEIA food categories have been previously published by USDA [15].

In the current analyses, each grain food and all other foods reported in WWEIA/NHANES is placed in one of the mutually-exclusive food subcategories by linking each food code contained in FNDDS to one WWEIA category [15]. Mixed grain dishes were separated from the main grains group as a food subcategory, and were included in the current analyses. In the present analyses, the combined NHANES dataset sample included 4522 male and female participants, $\geq 51$ years of age, who had reliable and complete 24-h dietary intake data from WWEIA. Trained individuals complete the 24-h dietary recalls using USDA's dietary data collection instrument, the Automated Multiple-Pass Method, which includes detailed descriptions of all food and amounts consumed by subjects. While two days of 24-h dietary recalls are collected in WWEIA, the current analysis used Day 1 data as this represents the in-person data collection in the Mobile Examination Center [16].

\section{Methods and Statistical Analysis}

All statistical analyses were performed using SAS software (Version 9.2, SAS Institute, Cary, NC, USA). SAS PROC SURVEYMEANS was used for all statistical calculations, including means and percentages. Survey weights were used to generate nationally-representative estimates for U.S. adults, which were also adjusted for the complex sample design of NHANES. Mean and standard errors of energy, macronutrients and micronutrients for the daily total diet and from food groups were determined (nutrients from dietary supplements were not considered). Percentages of total energy and nutrient intake contributed from food sources were tabulated by ranked order.

\section{Results}

Food sources of energy and nutrients for main food groups are shown in Tables 1-21, which appear in this article. Food sources of energy and nutrients for USDA food subcategories are shown in Supplemental Tables S1-S19 and are available online. All foods are ranked based on energy or nutrient contribution in the total diet. Only values $>1.0 \%$ contribution to the total diet are reported so as not to consider inconsequential sources.

\subsection{Main Food Groups and Subcategory Food Groups: Energy Contributions}

Energy contribution of main food groups and subcategory of foods groups are presented in Tables 1 and 2. The greatest main food group contributors of energy in the diet are mixed dishes $(19.3 \%)$, snacks and sweets $(17.0 \%)$, protein foods $(16.2 \%)$, grains $(14.1 \%)$ and non-alcoholic beverages $(7.2 \%)$ (Table 1). When considering subcategories of food groups, the top five ranking foods for energy contribution were sweet bakery products $(7.8 \%)$, breads, rolls, and tortillas $(7.6 \%)$, plant-based protein foods $(4.4 \%)$, alcoholic beverages $(4.3 \%)$ and grain-based mixed dishes $(4.2 \%)$. Other grain-based foods providing $\leq 5 \%$ of energy in the total diet included, sandwiches $(3.1 \%)$, ready-to-eat cereals $(2.4 \%)$, 
pizza $(2.3 \%)$, quick breads and bread products $(1.6 \%)$, cooked grains $(1.5 \%)$ crackers $(1.2 \%)$, and cooked cereals $(1.1 \%)$ (Table 2$)$.

Table 1. Main food group sources of energy ( $\mathrm{kcal}$ ) contribution in the total U.S. diet, adults $\geq 51$ years old $(n=4522$; gender combined; daily intake data; National Health and Nutrition Examination Survey. (NHANES) 2011-2014; main food groups contributing $<1.0 \%$ not reported; data are Day 1 intakes).

\begin{tabular}{ccc}
\hline Main Food Group & Rank & \% Energy Contribution \\
\hline Mixed Dishes & 1 & 19.34 \\
Snacks and Sweets & 2 & 16.97 \\
Protein Foods & 3 & 16.15 \\
Grains & 4 & 14.06 \\
Beverages, Nonalcoholic & 5 & 7.16 \\
Milk and Dairy & 6 & 6.47 \\
Vegetables & 7 & 5.94 \\
Alcoholic Beverages & 8 & 4.34 \\
Fats and Oils & 9 & 3.88 \\
Fruit & 10 & 3.17 \\
Sugars & 11 & 1.37 \\
\hline
\end{tabular}

Table 2. Subcategory food group sources of energy (kcal) contribution in the total U.S. diet, adults $\geq 51$ years old ( $n=4522$; gender combined; daily intake data; NHANES 2011-2014; food subcategories contributing $<1.0 \%$ not reported; data are Day 1 intakes).

\begin{tabular}{|c|c|c|}
\hline Food Group & Rank & $\%$ Energy Contribution \\
\hline Sweet Bakery Products & 1 & 7.77 \\
\hline Breads, Rolls, Tortillas & 2 & 7.58 \\
\hline Plant-based Protein Foods & 3 & 4.40 \\
\hline Alcoholic Beverages & 4 & 4.34 \\
\hline Mixed Dishes-Grain-based & 5 & 4.22 \\
\hline Mixed Dishes-Meat, Poultry, Fish & 6 & 4.12 \\
\hline Sweetened Beverages & 7 & 4.00 \\
\hline Fats and Oils & 8 & 3.88 \\
\hline Fruits & 9 & 3.17 \\
\hline White Potatoes & 10 & 3.16 \\
\hline Mixed Dishes - Sandwiches & 11 & 3.13 \\
\hline Poultry & 12 & 3.04 \\
\hline Savory Snacks & 13 & 3.03 \\
\hline Milk & 14 & 2.81 \\
\hline Vegetables, excluding Potatoes & 15 & 2.77 \\
\hline Mixed Dishes-Mexican & 16 & 2.74 \\
\hline Other Desserts & 17 & 2.66 \\
\hline Meats & 18 & 2.60 \\
\hline Cured Meats/Poultry & 19 & 2.47 \\
\hline Ready-to-Eat Cereals & 20 & 2.35 \\
\hline Mixed Dishes_Pizza & 21 & 2.26 \\
\hline Cheese & 22 & 2.22 \\
\hline Eggs & 23 & 2.07 \\
\hline Candy & 24 & 2.02 \\
\hline Quick Breads and Bread Products & 25 & 1.60 \\
\hline Seafood & 26 & 1.57 \\
\hline Mixed Dishes-Soups & 27 & 1.56 \\
\hline Coffee and Tea & 28 & 1.54 \\
\hline $100 \%$ Juice & 29 & 1.52 \\
\hline Cooked Grains & 30 & 1.46 \\
\hline Sugars & 31 & 1.37 \\
\hline Mixed Dishes-Asian & 32 & 1.31 \\
\hline Crackers & 33 & 1.15 \\
\hline Cooked Cereals & 34 & 1.07 \\
\hline
\end{tabular}




\subsection{Main Food Groups: 2015-2020 Dietary Guidelines for Americans Shortfall Nutrients}

3.2.1. 2015-2020 Dietary Guidelines Nutrients of Public Health Concern: Dietary Fiber, Calcium, Vitamin D, and Potassium

The highest ranking (i.e., $\geq 10 \%$ ) main food group contributors, providing $94.31 \%$ of dietary fiber in the diet, were grains $(23.1 \%)$, mixed dishes $(19.0 \%)$, vegetables $(18.3 \%)$, fruit $(11.7 \%)$, snacks and sweets $(11.7 \%)$, and protein foods $(10.6 \%)$ (Table 3$)$. The top ranking main food group contributors for calcium, providing $62.9 \%$ of calcium in the diet, were milk and dairy $(32.8 \%)$, mixed dishes $(16.9 \%)$, and grains $(13.2 \%)$ (Table 4$)$. When considering vitamin $\mathrm{D}$, the highest ranking main food group contributors providing $71.0 \%$ of vitamin $\mathrm{D}$ in the diet were milk and dairy $(40 \%)$ and protein foods $(31.0 \%)$ (Table 5). The largest main food group contributors of potassium were protein foods $(16.8 \%)$, mixed dishes (16.3\%), non-alcoholic beverages (16.2\%), and vegetables (14.7\%); collectively, these main food groups contributed $64.0 \%$ of potassium in the diet (Table 6).

Table 3. Food sources of dietary fiber contribution in the total U.S. diet, adults $\geq 51$ years old ( $n=4522$; gender combined; daily intake data; NHANES 2011-2014; main food groups contributing $<1.0 \%$ not reported; data are Day 1 intakes).

\begin{tabular}{ccc}
\hline Main Food Group & Rank & \% Fiber Contribution \\
\hline Grains & 1 & 23.12 \\
Mixed Dishes & 2 & 18.95 \\
Vegetables & 3 & 18.25 \\
Fruit & 4 & 11.70 \\
Snacks and Sweets & 5 & 11.69 \\
Protein Foods & 6 & 10.61 \\
Beverages, Nonalcoholic & 7 & 2.38 \\
Condiments and Sauces & 8 & 1.97 \\
\hline
\end{tabular}

Table 4. Food sources of calcium contribution in the total U.S. diet, adults $\geq 51$ years old ( $n=4522$; gender combined; daily intake data; NHANES 2011-2014; main food groups contributing $<1.0 \%$ not reported; data are Day 1 intakes).

\begin{tabular}{ccc}
\hline Main Food Group & Rank & $\begin{array}{c}\text { \% Calcium } \\
\text { Contribution }\end{array}$ \\
\hline Milk and Dairy & 1 & 32.77 \\
Mixed Dishes & 2 & 16.86 \\
Grains & 3 & 13.24 \\
Snacks and Sweets & 4 & 8.00 \\
Beverages, Nonalcoholic & 5 & 7.69 \\
Water & 6 & 6.33 \\
Protein Foods & 7 & 5.90 \\
Vegetables & 8 & 4.95 \\
Fruit & 9 & 1.22 \\
\hline
\end{tabular}

Table 5. Food sources of vitamin D (D2 + D3) contribution in the total U.S. diet, adults $\geq 51$ years old ( $n=4522$; gender combined; daily intake data; NHANES 2011-2014; DFE = dietary folate equivalents; main food groups contributing $<1.0 \%$ not reported; data are Day 1 intakes).

\begin{tabular}{ccc}
\hline Main Food Group & Rank & \% Vitamin D Contribution \\
\hline Milk and Dairy & 1 & 40.06 \\
Protein Foods & 2 & 30.95 \\
Grains & 3 & 9.33 \\
Mixed Dishes & 4 & 8.19 \\
Beverages, Nonalcoholic & 5 & 5.71 \\
Snacks and Sweets & 6 & 2.14 \\
Vegetables & 7 & 1.56 \\
Fats and Oils & 8 & 1.25 \\
\hline
\end{tabular}


Table 6. Food sources of potassium contribution in the total U.S. diet, adults $\geq 51$ years old $(n=4522$; gender combined; daily intake data; NHANES 2011-2014; main food groups contributing $<1.0 \%$ not reported; data are Day 1 intakes).

\begin{tabular}{ccc}
\hline Main Food Group & Rank & \% Potassium Contribution \\
\hline Protein Foods & 1 & 16.75 \\
Mixed Dishes & 2 & 16.32 \\
Beverages, Nonalcoholic & 3 & 16.17 \\
Vegetables & 4 & 14.73 \\
Milk and Dairy & 5 & 9.05 \\
Snacks and Sweets & 6 & 8.01 \\
Fruit & 7 & 7.18 \\
Grains & 8 & 6.66 \\
Alcoholic Beverages & 9 & 2.18 \\
Condiments and Sauces & 10 & 1.60 \\
\hline
\end{tabular}

3.2.2. 2015-2020 Dietary Guidelines Shortfall Nutrients: Folate, Iron, Magnesium, Vitamin A, Vitamin E, and Vitamin C

The largest ranking (i.e., $\geq 10 \%$ ) main food group contributors of dietary folate providing $60.3 \%$ of folate in the diet, were grains (40.5\%), and mixed dishes (19.9\%) (Table 7). The top ranking main food group contributors for iron, providing $83.4 \%$ of iron in the diet, were grains $(37.8 \%)$, mixed dishes $(19.6 \%)$, protein foods $(14.4 \%)$, and snacks and sweets (11.7\%) (Table 8$)$. For magnesium, the highest ranking main food group contributors providing $59.5 \%$ of magnesium in the diet were protein foods $(17.7 \%)$, grains $(15.0 \%)$, mixed dishes $(14.6 \%)$ and non-alcoholic beverages $(12.2 \%)$ (Table 9$)$. The largest main food group contributors of vitamin A were vegetables (23.0\%), milk and dairy (15.9\%), mixed dishes $(14.2 \%)$, grains $(12.9 \%)$, and protein foods $(11.6 \%)$, collectively, providing $77.7 \%$ of vitamin $\mathrm{A}$ in the diet (Table 10). The greatest main food group sources of vitamin E, contributing $78.3 \%$ of vitamin $\mathrm{E}$ in the diet, were protein foods $(22.9 \%)$, mixed dishes $(17.4 \%)$, snacks and sweets $(16.1 \%)$, vegetables $(11.4 \%)$, and grains $(10.5 \%)$ (Table 11$)$. The top three main food group sources of vitamin C were non-alcoholic beverages (36.0\%), vegetables $(25.6 \%)$, and fruit $(18.2 \%)$, contributing a total of $79.8 \%$ of all vitamin $C$ in the total daily diet (Table 12 ).

Table 7. Food sources of folate, DFE, contribution in the total U.S. diet, adults $\geq 51$ years old ( $n=4522$; gender combined; daily intake data; NHANES 2011-2014; DFE = dietary folate equivalents; main food groups contributing $<1.0 \%$ not reported; data are Day 1 intakes).

\begin{tabular}{ccc}
\hline Main Food Group & Rank & \% Folate, DFE Contribution \\
\hline Grains & 1 & 40.48 \\
Mixed Dishes & 2 & 19.85 \\
Snacks and Sweets & 3 & 9.69 \\
Vegetables & 4 & 9.46 \\
Protein Foods & 5 & 7.39 \\
Beverages, Nonalcoholic & 6 & 6.21 \\
Fruit & 7 & 2.22 \\
Milk and Dairy & 8 & 2.06 \\
Alcoholic Beverages & 9 & 1.39 \\
\hline
\end{tabular}


Table 8. Food sources of iron contribution in the total U.S. diet, adults $\geq 51$ years old $(n=4522$; gender combined; daily intake data; NHANES 2011-2014; main food groups contributing $<1.0 \%$ not reported; data are Day 1 intakes).

\begin{tabular}{ccc}
\hline Main Food Group & Rank & \% Iron Contribution \\
\hline Grains & 1 & 37.78 \\
Mixed Dishes & 2 & 19.61 \\
Protein Foods & 3 & 14.37 \\
Snacks and Sweets & 4 & 11.67 \\
Vegetables & 5 & 5.98 \\
Beverages, Nonalcoholic & 6 & 4.75 \\
Fruit & 7 & 1.72 \\
Milk and Dairy & 8 & 1.17 \\
Condiments and Sauces & 9 & 1.01 \\
\hline
\end{tabular}

Table 9. Food sources of magnesium contribution in the total U.S. diet, adults $\geq 51$ years old $(n=4522$; gender combined; daily intake data; NHANES 2011-2014; main food groups contributing $<1.0 \%$ not reported data are Day 1 intakes).

\begin{tabular}{ccc}
\hline Main Food Group & Rank & \% Magnesium Contribution \\
\hline Protein Foods & 1 & 17.69 \\
Grains & 2 & 15.00 \\
Mixed Dishes & 3 & 14.59 \\
Beverages, Nonalcoholic & 4 & 12.19 \\
Snacks and Sweets & 5 & 9.83 \\
Vegetables & 6 & 9.13 \\
Milk and Dairy & 7 & 7.15 \\
Water & 8 & 4.62 \\
Fruit & 9 & 4.20 \\
Alcoholic Beverages & 10 & 3.23 \\
Condiments and Sauces & 11 & 1.14 \\
\hline
\end{tabular}

Table 10. Food sources of vitamin A, retinol activity equivalents (RAE), contribution in the total U.S. diet, adults $\geq 51$ years old ( $n=4522$; gender combined; daily intake data; NHANES 2011-2014; main food groups contributing $<1.0 \%$ not reported; data are Day 1 intakes).

\begin{tabular}{ccc}
\hline Main Food Group & Rank & \% Vitamin A, RAE Contribution \\
\hline Vegetables & 1 & 23.01 \\
Milk and Dairy & 2 & 15.94 \\
Mixed Dishes & 3 & 14.19 \\
Grains & 4 & 12.93 \\
Protein Foods & 5 & 11.60 \\
Snacks and Sweets & 6 & 7.19 \\
Beverages, Nonalcoholic & 7 & 6.02 \\
Fats and Oils & 8 & 5.28 \\
Fruit & 9 & 2.16 \\
\hline
\end{tabular}


Table 11. Food sources of vitamin E, as alpha-tocopherol, contribution in the total U.S. diet, adults $\geq 51$ years old ( $n=4522$; gender combined; daily intake data; NHANES 2011-2014; main food groups contributing $<1.0 \%$ not reported; data are Day 1 intakes).

\begin{tabular}{ccc}
\hline Main Food Group & Rank & \% Vitamin E Contribution \\
\hline Protein Foods & 1 & 22.88 \\
Mixed Dishes & 2 & 17.38 \\
Snacks and Sweets & 3 & 16.11 \\
Vegetables & 4 & 11.44 \\
Grains & 5 & 10.47 \\
Fats and Oils & 6 & 7.37 \\
Beverages, Nonalcoholic & 7 & 5.06 \\
Fruit & 8 & 2.63 \\
Milk and Dairy & 9 & 2.57 \\
Condiments and Sauces & 10 & 2.48 \\
\hline
\end{tabular}

Table 12. Food sources of vitamin C contribution in the total U.S. diet, adults $\geq 51$ years old ( $n=4522$; gender combined; daily intake data; NHANES 2011-2014; main food groups contributing $<1.0 \%$ not reported; data are Day 1 intakes).

\begin{tabular}{ccc}
\hline Main Food Group & Rank & \% Vitamin C Contribution \\
\hline Beverages, Nonalcoholic & 1 & 35.98 \\
Vegetables & 2 & 25.60 \\
Fruit & 3 & 18.22 \\
Mixed Dishes & 4 & 9.01 \\
Grains & 5 & 3.39 \\
Snacks and Sweets & 6 & 2.50 \\
Condiments and Sauces & 7 & 1.98 \\
Protein Foods & 8 & 1.07 \\
\hline
\end{tabular}

\subsection{Subcategory Food Groups: 2015-2020 Dietary Guidelines for Americans' Shortfall Nutrients}

3.3.1. 2015-2020 Dietary Guidelines Nutrients of Public Health Concern: Dietary Fiber, Calcium, Vitamin D, and Potassium

The top ranking (i.e., $\geq 10 \%$ ) subcategory foods, collectively providing $37.4 \%$ of dietary fiber to the total diet, were vegetables, excluding potatoes $(13.6 \%)$, breads, rolls and tortillas (12.0\%) and fruits $(11.7 \%)$ (Table S1). When considering calcium, two food groups contributed approximately a quarter of all calcium in the diet $(27.5 \%)$ milk $(15.7 \%)$ and cheese $(11.8 \%)$ (Table S2). When considering vitamin D (Table S3), the highest ranking subcategory contributors providing $47.4 \%$ of vitamin D in the diet were milk (30.1\%) and seafood (17.4\%). While no one food item provided $\geq 10 \%$ of potassium in the total diet, coffee, vegetables (excluding potatoes), fruits, milk, white potatoes, meat/poultry/fish mixed dishes, plant-based protein foods, $100 \%$ juice, breads, rolls, and tortillas, and poultry were the top ten ranking contributors, collectively providing $57.3 \%$ of all potassium in the diet (Table S4).

3.3.2. 2015-2020 Dietary Guidelines Shortfall Nutrients: Folate, Iron, Magnesium, Vitamin A, Vitamin E, and Vitamin C

Two grain food groups were the two largest contributors (i.e., $\geq 10 \%$ ) of dietary folate as Dietary Folate Equivalents (DFE) providing 34.4\% of folate in the diet, namely ready-to-eat cereals $(21.0 \%)$, and breads, rolls and tortillas (13.4\%) (Table S5). Similarly, ready-to-eat cereals, and breads, rolls, and tortillas provided nearly one-third of all iron in the total diet (30.8\%) (Table S6). While no one food item provided $\geq 10 \%$ of all magnesium in the total diet, plant-based protein foods, breads, rolls, and tortillas, coffee and tea, vegetables (excluding potatoes), plain water, milk, fruits, ready-to-eat cereals, meat/poultry/fish mixed dishes, and grain-based mixed dishes were the top ten ranking contributors, collectively providing $53.6 \%$ of all magnesium in the diet (Table S7). Only vegetables, 
excluding potatoes, provided $\geq 10 \%$ of vitamin A in the total diet (21.7\%). An additional $47.2 \%$ of all vitamin A in the diet was providing by the following nine foods: milk, ready-to-eat cereals, fats and oils, eggs, meats, cheese, desserts, meat/poultry/fish mixed dishes, and grain-based mixed dishes (Table S8). The top ten ranking foods for vitamin E contributed $61.9 \%$ (Table S9) of vitamin E in the diet, however, the category of plant-based protein foods was the only food source to provide $\geq 10 \%$ of all vitamin E (12.7\%). The top three ranking foods for vitamin C (Table S10) provided $65.4 \%$ of all vitamin C in the diet: $100 \%$ juice $(25.4 \%)$, vegetables, excluding potatoes $(21.8 \%)$, and fruits $(18.2 \%)$.

\subsection{Main Food Groups: Total Fat, Saturated Fat, Added Sugars and Sodium Contributions}

Protein foods, mixed dishes, snacks and sweets were the source for nearly $77 \%$ of total fat in the diet (Table 13). Mixed dishes, snacks and sweets, protein foods, milk and dairy, and fats and oils contributed $88 \%$ of all saturated fat in the total diet (Table 14). Two main food groups, non-alcoholic beverages, and snacks and sweets contributed nearly $66 \%$ of added sugars in the diet (Table 15). The three largest main food group sources of sodium in the total diet were mixed dishes $(29.2 \%)$, protein foods (22.7\%), and grains (14.1\%) (Table 16).

Table 13. Food sources of total fat contribution in the total U.S. diet, adults $\geq 51$ years old $(n=4522$; gender combined; daily intake data; NHANES 2011-2014; main food groups contributing $<1.0 \%$ not reported; data are Day 1 intakes).

\begin{tabular}{ccc}
\hline Main Food Group & Rank & \% Total Fat Contribution \\
\hline Protein Foods & 1 & 25.31 \\
Mixed Dishes & 2 & 22.52 \\
Snacks and Sweets & 3 & 19.52 \\
Fats and Oils & 4 & 9.57 \\
Milk and Dairy & 5 & 7.51 \\
Vegetables & 6 & 6.35 \\
Grains & 7 & 6.27 \\
Condiments and Sauces & 8 & 1.41 \\
Beverages, Nonalcoholic & 9 & 1.02 \\
\hline
\end{tabular}

Table 14. Food sources of saturated fat contribution in the total U.S. diet, adults $\geq 51$ years old ( $n=4522$; gender combined; daily intake data; NHANES 2011-2014; main food groups contributing $<1.0 \%$ not reported; data are Day 1 intakes).

\begin{tabular}{ccc}
\hline Main Food Group & Rank & \% Saturated Fat Contribution \\
\hline Mixed Dishes & 1 & 23.47 \\
Snacks and Sweets & 2 & 21.18 \\
Protein Foods & 3 & 20.19 \\
Milk and Dairy & 4 & 14.08 \\
Fats and Oils & 5 & 9.52 \\
Grains & 6 & 4.58 \\
Vegetables & 7 & 4.44 \\
Condiments and Sauces & 8 & 1.32 \\
\hline
\end{tabular}


Table 15. Food sources of added sugars contribution in the total U.S. diet, adults $\geq 51$ years old ( $n=4522$; gender combined; daily intake data; NHANES 2011-2014; main food groups contributing $<1.0 \%$ not reported; data are Day 1 intakes).

\begin{tabular}{ccc}
\hline Main Food Group & Rank & \% Added Sugar Contribution \\
\hline Beverages, Nonalcoholic & 1 & 33.25 \\
Snacks and Sweets & 2 & 32.69 \\
Sugars & 3 & 9.09 \\
Grains & 4 & 8.90 \\
Milk and Dairy & 5 & 3.90 \\
Fats and Oils & 6 & 2.78 \\
Mixed Dishes & 7 & 2.71 \\
Protein Foods & 8 & 1.63 \\
Fruit & 9 & 1.43 \\
Alcoholic Beverages & 10 & 1.21 \\
\hline
\end{tabular}

Table 16. Food sources of sodium contribution in the total U.S. diet, adults $\geq 51$ years old $(n=4522$; gender combined; daily intake data; NHANES 2011-2014; main food groups contributing $<1.0 \%$ not reported; data are Day 1 intakes).

\begin{tabular}{ccc}
\hline Main Food Group & Rank & \% Sodium Contribution \\
\hline Mixed Dishes & 1 & 29.19 \\
Protein Foods & 2 & 22.68 \\
Grains & 3 & 14.13 \\
Snacks and Sweets & 4 & 8.57 \\
Vegetables & 5 & 8.10 \\
Milk and Dairy & 6 & 5.64 \\
Condiments and Sauces & 7 & 4.31 \\
Fats and Oils & 8 & 3.40 \\
Beverages, Nonalcoholic & 9 & 2.23 \\
Water & 10 & 1.14 \\
\hline
\end{tabular}

\subsection{Subcategory Food Groups: Total Fat, Saturated Fat, Added Sugars and Sodium Contributions}

While no one subcategory of food contributed $\geq 10 \%$ of all total fat (Table S11) in the diet, the top ten food sources collectively provided $56.9 \%$ of total fat, with fats and oils and sweet bakery products being the largest contributors. Likewise, while fats and oils, and sweet bakery products were the largest contributors of saturated fat ( $9.5 \%$ and $9.2 \%$, respectively), no food item contributed $\geq 10 \%$ of all saturated fat in the diet (Table S12). Two food groups provided nearly half of all added sugars in the total diet (Table S13) - sweetened beverages (25.0\%) and sweet bakery products $(17.2 \%)$. Similar to total fat and saturated fat, there was no food group that provided $\geq 10 \%$ of all sodium in the diet (Table S14). The top ten foods, collectively contributing $53.6 \%$ of all sodium in the total diet, were breads, rolls, and tortillas, cured meats/poultry, meat/poultry/fish mixed dishes, soups, grain-based mixed dishes, vegetables (excluding potatoes), poultry, condiments and sauces, sandwiches, and sweet bakery products.

\subsection{Main Food Groups: Thiamin, Niacin, Riboflavin, Vitamin B6, Vitamin B12}

Four main food groups contribute nearly $78 \%$ of all thiamin in the diet, with the leading main food group being grain foods, providing $32.6 \%$ of thiamin in the total diet (Table 17). For niacin, the top three contributors in the diet were protein foods $(29.1 \%)$, mixed dishes $(22.6 \%)$ and grains $(22.5 \%)$, collectively providing nearly $75 \%$ of niacin in the diet (Table 18). The top five contributors of riboflavin, collectively providing $81.2 \%$ of riboflavin were non-alcoholic beverages, grains, milk and dairy, protein foods, and mixed dishes (Table 19). Protein foods, grains, mixed dishes and vegetables were the largest contributors of vitamin B6 (72.7\%) (Table 20), while protein foods, mixed dishes, milk and dairy, and grains contributed nearly $90 \%$ of all vitamin B12 in the diet (Table 21). 
Table 17. Food sources of thiamin contribution in the total U.S. diet, adults $\geq 51$ years old ( $n=4522$; gender combined; daily intake data; NHANES 2011-2014; main food groups contributing $<1.0 \%$ not reported; data are Day 1 intakes).

\begin{tabular}{ccc}
\hline Main Food Group & Rank & \% Thiamin Contribution \\
\hline Grains & 1 & 32.56 \\
Mixed Dishes & 2 & 21.77 \\
Protein Foods & 3 & 13.60 \\
Snacks and Sweets & 4 & 10.06 \\
Beverages, Nonalcoholic & 5 & 6.60 \\
Vegetables & 6 & 6.55 \\
Milk and Dairy & 7 & 4.16 \\
Fruit & 8 & 2.30 \\
\hline
\end{tabular}

Table 18. Food sources of niacin contribution in the total U.S. diet, adults $\geq 51$ years old ( $n=4522$; gender combined; daily intake data; NHANES 2011-2014; main food groups contributing $<1.0 \%$ not reported; data are Day 1 intakes).

\begin{tabular}{ccc}
\hline Main Food Group & Rank & \% Niacin Contribution \\
\hline Protein Foods & 1 & 29.11 \\
Mixed Dishes & 2 & 22.57 \\
Grains & 3 & 22.52 \\
Snacks and Sweets & 4 & 7.06 \\
Beverages, Nonalcoholic & 5 & 6.77 \\
Vegetables & 6 & 5.25 \\
Alcoholic Beverages & 7 & 2.43 \\
Fruit & 8 & 1.66 \\
\hline
\end{tabular}

Table 19. Food sources of riboflavin contribution in the total U.S. diet, adults $\geq 51$ years old $(n=4522$; gender combined; daily intake data; NHANES 2011-2014; main food groups contributing $<1.0 \%$ not reported; data are Day 1 intakes).

\begin{tabular}{ccc}
\hline Main Food Group & Rank & \% Riboflavin Contribution \\
\hline Beverages, Nonalcoholic & 1 & 17.85 \\
Grains & 2 & 17.08 \\
Milk and Dairy & 3 & 15.95 \\
Protein Foods & 4 & 15.64 \\
Mixed Dishes & 5 & 14.70 \\
Snacks and Sweets & 6 & 8.94 \\
Vegetables & 7 & 4.18 \\
Fruit & 8 & 1.95 \\
Alcoholic Beverages & 9 & 1.51 \\
\hline
\end{tabular}

Table 20. Food sources of vitamin B6 contribution in the total U.S. diet, adults $\geq 51$ years old ( $n=4522$; gender combined; daily intake data; NHANES 2011-2014; main food groups contributing $<1.0 \%$ not reported; data are Day 1 intakes).

\begin{tabular}{ccc}
\hline Main Food Group & Rank & \% Vitamin B6 Contribution \\
\hline Protein Foods & 1 & 24.39 \\
Grains & 2 & 20.11 \\
Mixed Dishes & 3 & 16.51 \\
Vegetables & 4 & 11.70 \\
Beverages, Nonalcoholic & 5 & 5.99 \\
Fruit & 6 & 5.92 \\
Snacks and Sweets & 7 & 4.99 \\
Milk and Dairy & 8 & 3.55 \\
Alcoholic Beverages & 9 & 3.11 \\
Fats and Oils & 10 & 1.45 \\
Condiments and Sauces & 11 & 1.29 \\
\hline
\end{tabular}


Table 21. Food sources of vitamin B12 contribution in the total U.S. diet, adults $\geq 51$ years old $(n=4522$; gender combined; daily intake data; NHANES 2011-2014; main food groups contributing $<1.0 \%$ not reported; data are Day 1 intakes).

\begin{tabular}{ccc}
\hline Main Food Group & Rank & \% Vitamin B12 Contribution \\
\hline Protein Foods & 1 & 33.57 \\
Mixed Dishes & 2 & 19.01 \\
Milk and Dairy & 3 & 18.77 \\
Grains & 4 & 17.61 \\
Beverages, Nonalcoholic & 5 & 4.12 \\
Snacks and Sweets & 6 & 3.46 \\
Fats and Oils & 7 & 1.06 \\
Vegetables & 8 & 1.03 \\
\hline
\end{tabular}

\subsection{Subcategory Food Groups: Thiamin, Niacin, Riboflavin, Vitamin B6, Vitamin B12}

Two grain subcategories (i.e., breads, rolls and tortillas, and ready-to-eat cereals) were the largest contributors ( $\geq 10 \%$ for each food) of thiamin in the diet, collectively providing more than a quarter of thiamin in the total diet (Table S15). Breads, rolls, and tortillas were the only food to provide $\geq 10 \%$ of niacin in the total diet (Table S16). The top two contributors of riboflavin in the total diet were coffee and tea, and milk, collectively providing $23.8 \%$ of riboflavin in the diet (Table S17). Ready-to-eat cereals were the sole food subcategory to provide $\geq 10 \%$ of vitamin B6 in the total diet (Table S18), while meats, ready-to-eat cereals, and milk were the top-ranking subcategory of foods for vitamin B12, collectively providing $42.0 \%$ of all vitamin B12 in the total diet (Table S19).

\section{Discussion}

Americans of all ages are continuously not meeting nutrition recommendations even as previous [8] and current dietary guidelines [3] have identified shortfall and nutrients of public health concern. The current analyses focused on older American adults, as sources of energy and nutrients in this population remain limited. The present analyses determined the contribution of grain foods to energy and nutrients in older U.S. adults and ranked other food sources in the American diet, to provide an assessment of energy and nutrient contribution to the total diet. While all grain foods contributed $14 \%$ of all energy in the total diet, ranking as the 4th largest contributor of energy compared to 15 main food groups, grain foods can provide nutrient density to the American diet of older adults given they provide a greater percentage of nutrients than energy to the diet. The grain foods category ranked 2nd highest of 15 main food groups for daily dietary fiber, iron, folate, and magnesium, the 3rd largest main food group contributor for daily calcium intake $(13 \pm 0.4 \%)$ and 1 st for thiamin and niacin intake. When evaluating subcategories of foods, breads, rolls and tortillas provided $150 \pm 2.8 \mathrm{kcal} /$ day or $8 \%$ of all energy in the total diet, ranking as the 2nd largest contributor of energy compared to 46 food subcategories. The grain subcategory of breads, rolls and tortillas ranked 1st of 46 food subcategories for daily thiamin and niacin intake and 2nd for dietary fiber, iron, folate, and magnesium. Breads, rolls and tortillas ranked 3rd largest food subcategory contributor for daily calcium intake. Ready-to-eat cereals provided $47 \pm 2.6 \mathrm{kcal} /$ day or $2 \%$ of all energy in the total diet, ranking as the 20th largest contributor of energy compared to 46 food subcategories. All ready-to-eat cereals ranked 1st for daily iron, 1st for folate, 5th for dietary fiber, 3rd for niacin, 8th for magnesium, and 13th for calcium intake. When considering nutrients to limit as outlined by dietary guidance, main group of grains contributed $6.27 \%$ total fat, $4.58 \%$ saturated fat, $14.13 \%$ sodium and $8.9 \%$ added sugar.

Current and preceding dietary guidance $[3,8]$ for Americans recommend that half of all grains consumed be whole grains, with limitations placed on refined/enriched grain consumption. This may be promoting a perception that non-whole grain foods are not a component of a healthy dietary pattern, due to minimal or less nutrient density and increased caloric intake. The current analysis considered all foods consumed in the American diet, including all grain foods. Additionally, the current analysis did not separate whole- and refined-grain foods, as a key objective included determining the sources 
of energy and nutrients from all foods, and grain foods in particular, as currently consumed in the older American population. As such, the analysis showed that any perceptions that refined grain foods may be lacking in nutrient density are invalid. Indeed, certain grain foods (i.e., sweet bakery products) in the total diet can contribute nutrients to limit, including added sugar, sodium, and saturated fat and hence, these grain foods should be consumed in moderation and within caloric recommendations as designated by dietary guidance to promote public health. Nonetheless, the entire grain food category is the first, second, or third ranking category delivering dietary fiber, folate, iron, magnesium, calcium, and vitamin D-all nutrients identified as shortfall nutrients by the 2015-2020 DGA [3]. Similarly, further analysis with specific food subcategories within the grain category show that breads, rolls and tortillas, grain foods routinely consumed in the U.S. diet, are also first, second and/or third ranking foods for dietary fiber, folate, calcium, iron, magnesium, vitamin $\mathrm{A}$, for less than $8.5 \%$ of all calories and sodium, less than $3 \%$ of total fat, less than $2.5 \%$ of all saturated fat, and less than $3.5 \%$ of all added sugar in the total diet. Comparably, the current data provide sources of energy and nutrients data in the older American adult for ready-to-eat cereals, another commonly consumed grain food in the U.S. Ready-to-eat cereals are first, second, third, fourth, and/or fifth ranking foods for dietary fiber, folate, iron, vitamin $\mathrm{A}$, vitamin $\mathrm{D}$ for less than $2.5 \%$ of all calories and sodium, less than $1 \%$ of total fat, less than $0.5 \%$ of all saturated fat, and less than $4 \%$ of all added sugar in the total diet.

Many older Americans are not meeting recommendations for several key nutrients, many of which are contributed by grain food products. Thus, maintaining both whole- and refined/enriched grains, as part of a healthy dietary pattern, may prove to benefit nutrient intakes in a substantial portion of the U.S. older population. The 2015-2020 DGAs identified shortfall nutrients in all Americans 2 years of age, however, intake of shortfall nutrients can be even lower in older adults. A recent NHANES analysis examining food and supplement use in various age groups found significant differences in the proportion of the population with intakes below EAR. Adults $\geq 71$ years of age had a higher prevalence of inadequacy for calcium, magnesium, and most B vitamins compared to middle-aged and younger adults. Overall, when considering food consumption alone, the data showed that older adults had lower intake and higher prevalence of inadequacy for most nutrients relative to younger adults, with use of dietary supplements being associated with the greatest benefit in older adults [12]. An Irish cross-sectional analysis that explored dietary intakes of community-dwelling elderly individuals found that a substantial number of participants had inadequate intakes of several nutrients, including calcium, magnesium, vitamin D, folate, zinc and vitamin C [17]. U.S. national surveys of dietary intake consistently report all Americans, including older adults, do not meet recommendations for dietary fiber intake [18]. A review by Allen (2009) reported that in the U.S. and the United Kingdom, approximately $6 \%$ of adults $\geq 60$ years of age are vitamin B12 deficient, with prevalence of deficiency increasing as individuals age [19]. Another cross-sectional study of free-living New York city older adults participating in the Cardiovascular Health of Seniors and Built Environment Study, found that both genders fell short on recommended intake for fiber, calcium, magnesium, potassium, zinc, folate, and vitamins A, B6, C, D, and E, and in some instances, women had intakes for thiamin below the recommendations [20]. Similarly, a recent systematic review identified inadequate intake of vitamin $\mathrm{D}$, thiamin, riboflavin, calcium, magnesium, and selenium in community-dwelling older adults from North America, Europe, Australia and New Zealand [21] population.

The current analyses have a limitation inherent in observational research and has previously been reported in similar research designs. The results are dependent on self-reported dietary data for foods, which may involve study participants under- or over-estimating food consumption, leading to inaccuracies in energy and nutrient intakes. Data were also obtained using a 24-hour dietary recall, which relies on study participant memory, and while validated methods are used to gather the data, recall information is subject to inaccuracies and bias from memory challenges and other potential measurement errors experienced in epidemiological investigations [22]. Caution should be administered when comparing the current findings to previous studies, as food groupings can impact sources of energy and nutrient outcomes, specifically where there are differences in the level 
of aggregation (i.e., the number of food groups) or disaggregation methods used by researchers. A significant benefit of using NHANES data for the current analyses includes access to a large and nationally-representative dataset of adults of various age groups in the U.S. and corresponding food and nutrient intake data.

\section{Conclusions}

Although existing dietary guidance recommends an increase in shortfall nutrients and nutrients of public health concern, predominantly through food consumption, gaps in nutrient intakes persist in older U.S. adults. The current sources of energy and nutrients analyses show that cumulatively, a variety of grain foods, regularly consumed by older American adults, in addition to other nutrient-dense foods, including fruits and vegetables, help contribute to nutrient density in the total diet. Specifically, grain foods are contributors ( $\geq 10 \%$ in the total diet) of the 2015-2020 DGA under-consumed shortfall nutrients and nutrients of public health concern, including dietary fiber, folate, magnesium, calcium and iron, vitamin A, vitamin E, vitamin B12, niacin, and thiamin. The subcategory of breads, rolls and tortillas are contributors of daily thiamin, niacin, dietary fiber, folate, and iron, while ready-to-eat cereals contribute iron, folate, thiamin, vitamin B6, and vitamin B12. Based on the current data presented, encouraging certain grain food patterns in older U.S. adults, including selecting a mix of whole-, enriched- and fortified-grains, from breads, and cereals, may improve nutrient intakes and minimize gaps in shortfall nutrient intakes. In contrast, eliminating grains, especially refined grains, from the diet may lead to unintended nutrient intake consequences. Developing dietary strategies that are mindful of recommended caloric needs, while monitoring nutrients to limit (i.e., added sugars, sodium and saturated fat) can benefit from the inclusion of refined/enriched and whole grain selections in the U.S. diet.

Supplementary Materials: The following are available online at http:/ / www.mdpi.com/2072-6643/10/5/534/s1, Table S1: Subcategory food sources of dietary fiber contribution in the total U.S. diet, adults $\geq 51$ years old ( $n=4522$; gender combined; daily intake data; NHANES 2011-2014; subcategory groups contributing $<1.0 \%$ not reported; data are Day 1 intakes), Table S2: Subcategory food sources of calcium contribution in the total U.S. diet, adults $\geq 51$ years old ( $n=4522$; gender combined; daily intake data; NHANES 2011-2014; subcategory groups contributing $<1.0 \%$ not reported; data are Day 1 intakes), Table S3: Subcategory food sources of vitamin D (D2+D3) contribution in the total U.S. diet, adults $\geq 51$ years old ( $n=4522$; gender combined; daily intake data; NHANES 2011-2014; subcategory groups contributing $<1.0 \%$ not reported; data are Day 1 intakes), Table S4: Subcategory food sources of potassium contribution in the total U.S. diet, adults $\geq 51$ years old $(n=4522$; gender combined; daily intake data; NHANES 2011-2014; subcategory groups contributing $<1.0 \%$ not reported; data are Day 1 intakes), Table S5: Subcategory food sources of folate, DFE, contribution in the total U.S. diet, adults $\geq 51$ years old ( $n=4522$; gender combined; daily intake data; NHANES 2011-2014; subcategory groups contributing $<1.0 \%$ not reported; data are Day 1 intakes), Table S6: Subcategory food sources of iron contribution in the total U.S. diet, adults $\geq 51$ years old ( $n=4522$; gender combined; daily intake data; NHANES 2011-2014; subcategory groups contributing $<1.0 \%$ not reported; data are Day 1 intakes), Table S7: Subcategory food sources of magnesium contribution in the total U.S. diet, adults $\geq 51$ years old $(n=4522$; gender combined; daily intake data; NHANES 2011-2014; subcategory groups contributing $<1.0 \%$ not reported; data are Day 1 intakes), Table S8: Subcategory food sources of vitamin A, RAE, contribution in the total U.S. diet, adults $\geq 51$ years old $(n=4522$; gender combined; daily intake data; NHANES 2011-2014; subcategory groups contributing $<1.0 \%$ not reported; data are Day 1 intakes), Table S9: Subcategory food sources of vitamin E, as alpha tocopherol, contribution in the total U.S. diet, adults $\geq 51$ years old $(n=4522$; gender combined; daily intake data; NHANES 2011-2014; subcategory groups contributing $<1.0 \%$ not reported; data are Day 1 intakes), Table S10: Subcategory food sources of vitamin C contribution in the total U.S. diet, adults $\geq 51$ years old $(n=4522$; gender combined; daily intake data; NHANES 2011-2014; subcategory groups contributing <1.0\% not reported; data are Day 1 intakes), Table S11: Food sources of total fat contribution in the total U.S. diet, adults $\geq 51$ years old $(n=4522$; gender combined; daily intake data; NHANES 2011-2014; subcategory groups contributing $<1.0 \%$ not reported; data are Day 1 intakes), Table S12: Food sources of saturated fat contribution in the total U.S. diet, adults $\geq 51$ years old $(n=4522$; gender combined; daily intake data; NHANES 2011-2014; subcategory groups contributing $<1.0 \%$ not reported; data are Day 1 intakes), Table S13: Food sources of added sugar contribution in the total U.S. diet, adults $\geq 51$ years old ( $n=4522$; gender combined; daily intake data; NHANES 2011-2014; subcategory groups contributing $<1.0 \%$ not reported; data are Day 1 intakes), Table S14: Food sources of sodium contribution in the total U.S. diet, adults $\geq 51$ years old ( $n=4522$; gender combined; daily intake data; NHANES 2011-2014; subcategory groups contributing $<1.0 \%$ not reported; data are Day 1 intakes), Table S15: Food sources of thiamin contribution in the total U.S. diet, adults $\geq 51$ years old $(n=4522$; gender combined; daily intake data; NHANES 2011-2014; subcategory groups contributing <1.0\% not reported; data are Day 1 intakes), Table S16: Food sources 
of niacin contribution in the total U.S. diet, adults $\geq 51$ years old ( $n=4522$; gender combined; daily intake data; NHANES 2011-2014; subcategory groups contributing <1.0\% not reported; data are Day 1 intakes), Table S17: Food sources of riboflavin contribution in the total U.S. diet, adults $\geq 51$ years old $(n=4522$; gender combined; daily intake data; NHANES 2011-2014; subcategory groups contributing $<1.0 \%$ not reported; data are Day 1 intakes), Table S18: Food sources of vitamin B6 contribution in the total U.S. diet, adults $\geq 51$ years old $(n=4522$; gender combined; daily intake data; NHANES 2011-2014; subcategory groups contributing $<1.0 \%$ not reported; data are Day 1 intakes), Table S19: Food sources of vitamin B12 contribution in the total U.S. diet, adults $\geq 51$ years old ( $n=4522$; gender combined; daily intake data; NHANES 2011-2014; subcategory groups contributing $<1.0 \%$ not reported; data are Day 1 intakes).

Author Contributions: Y.P. and V.L.F., III collaborated on the intellectual conception and interpretation of the research; V.L.F., III collaborated on the intellectual conception and design of the research and conducted the final analyses; Y.P. drafted the manuscript and both Y.P. and V.L.F., III approved the final manuscript version of the present research.

Funding: The present research was funded and supported by the Grain Foods Foundation in Washington, D.C.

Conflicts of Interest: The authors declare no conflict of interest. The funding sponsors had no role in the design of the study; in the collection, analyses, or interpretation of data; in the writing of the manuscript, and in the decision to publish the results. Yanni Papanikolaou, as Vice-President of Nutritional Strategies provides food, nutrition and regulatory affairs consulting services for food and beverage companies and food-related associations and collaborates with Victor L. Fulgoni on NHANES analyses. Victor L. Fulgoni, III, as Senior Vice-President of Nutrition Impact, provides food and nutrition consulting services for food and beverage companies. Victor L. Fulgoni also conducts analyses of NHANES data for members of the food industry.

\section{References}

1. Papanikolaou, Y.; Fulgoni, V.L. Certain grain foods can be meaningful contributors to nutrient density in the diets of U.S. children and adolescents: Data from the National Health and Nutrition Examination Survey, 2009-2012. Nutrients 2017, 9, 160. [CrossRef] [PubMed]

2. Papanikolaou, Y.; Fulgoni, V.L. Grain foods are contributors of nutrient density for American adults and help close nutrient recommendation gaps: Data from the National Health and Nutrition Examination Survey, 2009-2012. Nutrients 2017, 9, 873. [CrossRef] [PubMed]

3. United States Department of Health and Human Services and United States Department of Agriculture. 2015-2020 Dietary Guidelines for Americans, 8th ed.; U.S. Government Printing Office: Washington, DC, USA, 2015. Available online: https:/ /health.gov/dietaryguidelines/2015/resources/2015-2020_Dietary_Guidelines.pdf (accessed on 7 February 2018).

4. Huth, P.J.; Fulgoni, V.L.; Keast, D.R.; Park, K.; Auestad, N. Major food sources of calories, added sugars, and saturated fat and their contribution to essential nutrient intakes in the U.S. diet: Data from the National Health and Nutrition Examination Survey (2003-2006). Nutr. J. 2013, 12, 116. [CrossRef] [PubMed]

5. Centers for Disease Control and Prevention. The State of Aging and Health in America 2013; Centers for Disease Control and Prevention, US Dept of Health and Human Services: Atlanta, GA, USA, 2013. Available online: https:/ / www.cdc.gov/aging/pdf/State-Aging-Health-in-America-2013.pdf (accessed on 2 February 2018).

6. Cody, M.M.; Stretch, T. Position of the Academy of Nutrition and Dietetics: Food and Nutrition for Older Adults: Promoting Health and Wellness. J. Acad. Nutr. Diet. 2012, 112, 1255-1277.

7. U.S. Department of Agriculture; U.S. Department of Health and Human Services (USDA/HHS). Scientific Report of the 2010 Dietary Guidelines Advisory Committee: Advisory Report to the Secretary of Health and Human Services and Secretary of Agriculture; USDA, Agricultural Research Service: Washington, DC, USA, 2010.

8. U.S. Department of Agriculture; U.S. Department of Health and Human Services. Dietary Guidelines for Americans, 2010, 7th ed.; U.S. Government Printing Office: Washington, DC, USA, 2010.

9. Keast, D.R.; Fulgoni, V.L.; Nicklas, T.A.; O'Neil, C.E. Food sources of energy and nutrients among children in the United States: NHANES 2003-2006. Nutrients 2013, 5, 283-301. [CrossRef] [PubMed]

10. O'Neil, C.E.; Keast, D.R.; Fulgoni, V.L.; Nicklas, T.A. Food sources of energy and nutrients among adults in the US: NHANES 2003-2006. Nutrients 2012, 4, 2097-2120. [CrossRef] [PubMed]

11. U.S. Department of Agriculture; U.S. Department of Health and Human Services (USDA/HHS). Scientific Report of the 2015 Dietary Guidelines Advisory Committee: Advisory Report to the Secretary of Health and Human Services and Secretary of Agriculture; USDA, Agricultural Research Service: Washington, DC, USA, 2015. Available online: https://ods.od.nih.gov/pubs/2015_DGAC_Scientific_Report.pdf (accessed on 31 January 2018). 
12. Blumberg, J.; Frei, B.; Fulgoni, V.L.; Weaver, C.M.; Zeisel, S.H. Contribution of dietary supplements to nutritional adequacy in various adult age groups. Nutrients 2017, 9, 1325. [CrossRef] [PubMed]

13. Centers for Disease Control and Prevention. National Center for Health Statistics. National Health and Nutrition Examination Survey. Analytic and Reporting Guidelines; Centers for Disease Control and Prevention: Atlanta, GA, USA, 2018. Available online: https://wwwn.cdc.gov/nchs/nhanes/analyticguidelines.aspx (accessed on 7 January 2018).

14. Ahuja, J.K.A.; Montville, J.B.; Omolewa-Tomobi, G.; Heendeniya, K.Y.; Martin, C.L.; Steinfeldt, L.C.; Anand, J.; Adler, M.E.; LaComb, R.P.; Moshfegh, A.J. USDA Food and Nutrient Database for Dietary Studies, 5.0-Documentation and User Guide; U.S. Department of Agriculture; Agricultural Research Service; Food Surveys Research Group: Beltsville, MD, USA, 2012.

15. United States Department of Agriculture. Agricultural Research Service. Food Service Research Group. Beltsville, MD, USA. Available online: https:/ / www.ars.usda.gov/northeast-area/beltsville-md-bhnrc/ beltsville-human-nutrition-research-center/food-surveys-research-group/docs/main-service-page/ (accessed on 23 February 2018).

16. United States Department of Agriculture; Agricultural Research Service; Food Service Research Group. What We Eat in America, National Health and Nutrition Examination Survey Overview, 2011-2014: Data Collection; United States Department of Agriculture, Agricultural Research Service: Beltsville, MD, USA, 2015. Available online: https:/ / www.ars.usda.gov/northeast-area/beltsville-md-bhnrc/beltsville-human-nutritionresearch-center/food-surveys-research-group/docs/wweianhanes-overview/ (accessed on 21 March 2018).

17. Power, S.E.; Jeffrey, I.B.; Ross, R.P.; Stanton, C.; O'Toole, P.W.; O'Connor, E.M.; Fitzgerald, G.F. Food and nutrient intake of Irish community-dwelling elderly subjects: Who is at Nutritional Risk? J. Nutr. Health Aging 2014, 18, 561-572. [CrossRef] [PubMed]

18. United States Department of Agriculture. Agricultural Research Service. Dietary Fiber (g): Usual Intakes from Food and Water, Compared to Adequate Intakes. What We Eat in America, NHANES 2003-2006; United States Department of Agriculture: Washington, DC, USA, 2007. Available online: https://www.ars.usda. gov / ARSUserFiles/80400530/pdf/0506/usual_nutrient_intake_dietary_fiber_2003-06.pdf (accessed on 31 January 2018).

19. Allen, L.H. How common is vitamin B-12 deficiency? Am. J. Clin. Nutr. 2009, 89, 693S-696S. [CrossRef] [PubMed]

20. Deierlein, A.L.; Morland, K.B.; Scanlin, K.; Wong, S.; Spark, A. Diet quality of urban older adults aged 60-99: The Cardiovascular Health of Seniors and Built Environment Study. J. Acad. Nutr. Diet. 2014, 114, $279-287$. [CrossRef] [PubMed]

21. Ter Borg, S.; Verlaan, S.; Hemsworth, J.; Mijnarends, D.M.; Schols, J.M.; Luiking, Y.C.; de Groot, L.C. Micronutrient intakes and potential inadequacies of community-dwelling older adults: A systematic review. Br. J. Nutr. 2015, 113, 1195-1206. [CrossRef] [PubMed]

22. Dwyer, J.; Picciano, M.F.; Raiten, D.J. Members of the Steering Committee. Collection of food and dietary supplement intake data: What we eat in America-NHANES. J. Nutr. 2003, 133, 590S-600S. [CrossRef] [PubMed]

(C) 2018 by the authors. Licensee MDPI, Basel, Switzerland. This article is an open access article distributed under the terms and conditions of the Creative Commons Attribution (CC BY) license (http://creativecommons.org/licenses/by/4.0/). 